\title{
Non-Neoplastic TdT-Positive Cells in Bone Marrow Trephines with Acute Myeloid Leukaemia before and after Treatment Express Myeloid Molecules
}

\author{
Korinna Jöhrens Aylin Franke Manfred Dietel Ioannis Anagnostopoulos \\ Institute of Pathology, Charité - Universitätsmedizin Berlin, Campus Mitte, Berlin, Germany
}

\author{
Key Words \\ Terminal deoxynucleotidyltransferase $\cdot$ Acute myeloid \\ leukaemia $\cdot$ Immunohistochemistry $\cdot$ Double \\ immunolabelling
}

\begin{abstract}
Objectives: Terminal deoxynucleotidyltransferase (TdT)positive cells in non-neoplastic bone marrow are regarded as haematogones and show a characteristic morphology and immunophenotype. During our routine bone marrow trephine analysis of patients with acute myeloid leukaemia (AML) before and after treatment, we observed the presence of TdT-positive cells lacking CD34, CD10 and B cell antigens. Methods: To verify the nature of these TdT-positive cells, we analyzed 140 paraffin-embedded and decalcified trephines from $67 \mathrm{AML}$ patients before and after therapy using double immunolabellings. Results: In $61 \%$ of the cases TdT-positive cells were present. After exclusion of neoplastic cells and haematogones, we identified that $16 \%$ of the cases harboured cells co-expressing myeloperoxidase and TdT, 15\% glycophorin C and TdT, 13\% CD117 and TdT and one case CD3 and TdT. These cells made up to $30 \%$ of the non-neoplastic TdT-positive cells. No differences in the number of TdT-positive cells before and after chemotherapy or stem cell transplantation could be observed. Conclusion: While the reason of TdT expression by non-neoplastic myeloid cells is unknown, their presence should be taken into account when evaluating such cases.

Copyright $\odot 2011$ S. Karger AG, Basel
\end{abstract}

\section{Introduction}

Terminal deoxynucleotidyltransferase (TdT) gene located at chromosome 10q23-q24 [1] encodes for a unique DNA polymerase that catalyzes the addition of non-templated $(\mathrm{N})$ nucleotides onto the free 3-prime-hydroxyl end of DNA primers [2]. The only known physiological function of TdT is the random addition of nucleotides to the $\mathrm{V}(\mathrm{D}) \mathrm{J}$ junctions of immunoglobulin heavy chain and $\mathrm{T}$ cell receptor gene rearrangements [3]. Consequently, the enzyme is present in the $\mathrm{B}$ and $\mathrm{T}$ cell precursors in bone marrow and thymus, as well as in the neoplasias and cell lines originating from these cells. TdT is additionally expressed in a proportion of acute myeloid leukaemia (AML) $[4,5]$.

In normal bone marrow trephines, TdT-positive cells are accepted as haematogones [6]. These cells show a high nuclear to cytoplasm ratio, homogeneously condensed nuclear chromatin with absent to inconspicuous nucleoli and variable nuclear size. A continuum of various lymphoid and B cell antigens are expressed by these cells, including CD34, CD45, CD19, CD20 and CD10 reflecting ongoing lymphoid maturation [7]. Haematogones are more abundant in children than in adults $[8$, 9]. Conditions of significant bone marrow suppression or B cell dysfunction in adults including multi-agent chemotherapy, bone marrow transplantation and infections are linked with increased haematogones [10]. Using an antibody panel including TdT in our daily diagnostic

\section{KARGER \\ Fax +4161306 1234 \\ E-Mail karger@karger.ch}

www.karger.com (c) 2011 S. Karger AG, Basel

1015-2008/11/0781-0035\$38.00/0

Accessible online at:

www.karger.com/pat
Korinna Jöhrens, MD

Institute of Pathology, Charité Campus Mitte

Charité - Universitätsmedizin Berlin

Charitéplatz 1, DE-10117 Berlin (Germany)

Tel. +49 30450536 026, Fax +4930450536 900, E-Mail korinna.joehrens@ charite.de 
routine, we could observe that a proportion of patients with AML have increased TdT-positive non-neoplastic cells at time of diagnosis as well as after therapy often without an additional expression of CD20, CD10 or CD19. Due to the fact that these patients have a highly suppressed bone marrow caused by AML or therapy, the question arose whether these cells might also be of myeloid origin. To clarify this question, we investigated the TdT-positive non-neoplastic cells systematically by double immunolabelling of paraffin-embedded bone marrow trephines coupling detection of TdT with CD3, CD10, CD19, glycophorin C, myeloperoxidase (MPO) and CD117.

\section{Patients and Methods}

For this retrospective investigation, 140 paraffin-embedded and decalcified bone marrow trephines of 67 patients with AML diagnosed from 2007 to March 2009 were retrieved from the archives of the Institute of Pathology, Charité Universitätsmedizin Berlin, Campus Mitte. All samples from 67 patients were anonymised. Forty-five patients had more than one biopsy (usually before and after therapy), while in the other 24 patients we had the possibility to investigate only one biopsy usually from the leukaemia manifestation and less often after therapy. Nineteen patients underwent stem cell transplantation (SCT), whereas the others were treated by chemotherapy. The AMLs were classified according to the WHO/FAB classifications after a combination of histological, immunophenotypical, fluorescence-activated cell sorting and cytogenetic analysis as follows: 15 patients were grouped into AML with minimal differentiation/M0, 14 into AML without maturation/M1, 18 into AML with maturation/ M2, 2 patients were verified as an acute promyelocytic leukaemia/M3, 4 patients as acute myelomonocytic leukaemia/M4, 3 patients as an acute monocytic/monoblastic leukaemia/M5 and 1 patient as acute erythroid leukaemia/M6. Ten cases were separated as secondary AML and one showed the features of AML with a translocation $\mathrm{t}(8 ; 21)$. Among the patients there were 43 males and 24 females. Ten patients were younger than 40 years at the time of diagnosis. After SCT the biopsies were performed at days 21-28.

Double labelling on the tissue sections was accomplished using two different polymer detection systems both obtained from Leica Microsystems: (1) the Bond Polymer Refine (a peroxidasebased detection reagent) and (2) the Bond Polymer AP Red (an alkaline phosphatase detection reagent). The stainings were performed in the Bond- $\max ^{\mathrm{TM}}$ immunostainer (Leica Microsystems) using the software-based double staining protocol. This multistep protocol initially performs an incubation of the first antibody (CD3, CD19, CD10, CD117, glycophorin C and myeloperoxidase) followed by the peroxidase-based detection system which uses diaminobenzidine for visualisation of the reaction product. Afterwards the sections became incubated with the TdT antibody. Then the alkaline phosphatase reagent was applied with visualisation of the reaction by means of the Fast Red substrate. Finally, the slides were counterstained with haematoxylin. The antibodies employed in this study were: TdT (clone SEN28), CD3 (clone LN10), CD10 (clone 56C6), CD19 (clone BT51E) all from Leica Microsystems, Novocastra Laboratories (Newcastle upon Tyne, UK), CD117 (polyclonal), glycophorin C (clone Ret40f) obtained from Dako (Glostrup, Denmark) and myeloperoxidase (polyclonal) obtained from Thermo-Fisher Scientific (Fremont, USA).

The percentage of positive cells was determined in the respective double labellings after counting of all labelled cells in each trephine. AML cells were identified not only on histomorphological features but also in combination with appropriate immunostains for CD34, CD117, myeloperoxidase, CD68 and glycophorin C. Non-neoplastic TdT-positive cells exhibited smaller nuclei than the AML cells as well as a different distribution (fig. 1a, b).

\section{Results}

\section{TdT-Positive Cells}

In $41(61 \%)$ of the investigated cases, TdT-positive cells made up $5-30 \%$ of the total cellularity. In 11 of these cases a variable proportion of the AML cells showed a co-expression of TdT. The AML cases with TdT-positive tumour cells were classified as minimally differentiated/M0 (1 case), without maturation/M1 (1 case), with maturation/M2 (4 cases), promyelocytic/M3 (2 cases), monoblastic/monocytic/M5 (1 case), secondary AML (2 cases).

In 6 cases an increase in the total number of TdT-positive cells could be observed after chemotherapy and in additional 8 cases after SCT. A decrease in non-neoplastic TdT-positive cells was found in 4 cases after chemotherapy and in 6 cases after SCT. The remaining cases did not show any variation in the total number of TdT-positive cells after therapy.

\section{CD19/TdT-Positive Cells}

Twenty-seven (40\%) of the cases contained a variable proportion (1-90\%) of CD19/TdT-positive cells (fig. 2a). This population increased in 5 cases after chemotherapy and in 6 cases after SCT. In 3 cases after chemotherapy and in 6 cases after SCT this population was highly diminished to absent.

\section{CD10/TdT-Positive Cells}

In $32(48 \%)$ of all investigated cases $5-80 \%$ of the nonneoplastic TdT-positive cells showed a co-expression of CD10 and TdT (fig. 2b). In 7 cases after chemotherapy and in 6 cases after SCT an increase in CD10/TdT+ cells could be observed, whereas in 4 cases after chemotherapy and 6 cases after SCT these cells were present in lower numbers than before. 


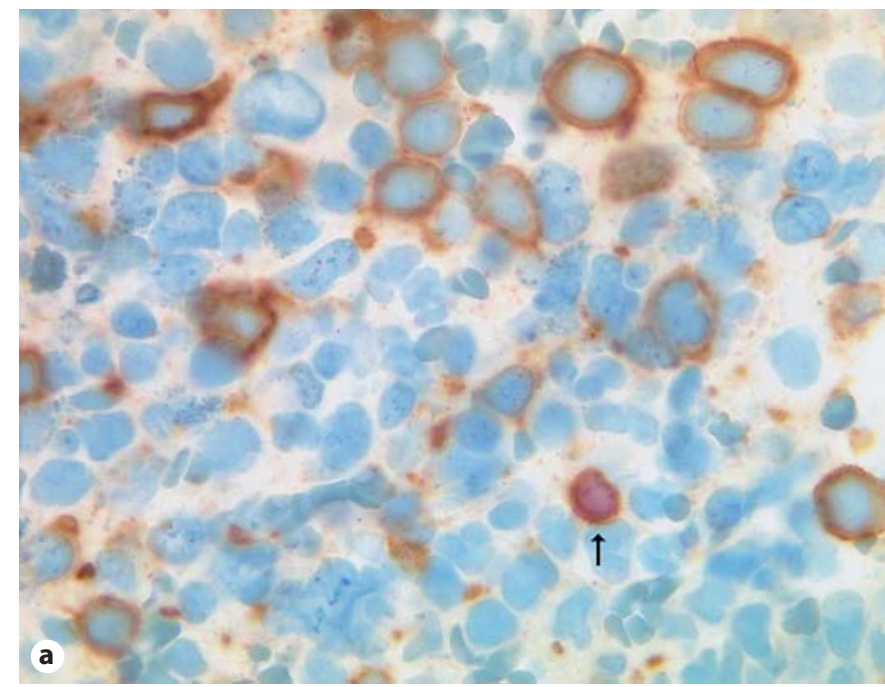

Fig. 1. Illustration of the different topography as well of the different nuclear size between neoplastic cells of acute leukaemia and non-neoplastic TdT-positive cells expressing myeloid antigens by using double immunolabellings in paraffin sections of decalcified bone marrow trephines. a In an acute myeloid leukaemia with maturation the TdT (brown nuclear stain) and CD117 (red cyto-

\section{CD117/TdT-Positive Cells}

In 9 cases (13\%), non-neoplastic TdT and CD117 coexpressing cells with smaller nuclei and different distribution than the AML population could be identified (fig. 1a). It is of importance to note that none of these AML cases contained TdT-positive cells. These made up $10-80 \%$ of the non-neoplastic TdT-positive cell population and expressed TdT with a variable intensity (fig. 2c). In 2 of these cases the number of TdT/CD117-positive non-neoplastic cells increased after SCT, in further 5 cases the number of these cells decreased after chemotherapy (4 cases) or after SCT (1 case).

\section{Myeloperoxidase/TdT-Positive Cells}

Eleven cases (16\%) harboured non-neoplastic cells exhibiting a co-expression of myeloperoxidase and TdT (fig. 1b, 2d). These cells made up to $30 \%$ of the TdT-positive non-neoplastic cells. In 1 of these cases after chemotherapy and in further 4 after SCT the number of these cells increased. In 5 cases ( 2 after chemotherapy and 3 after SCT) this cell population decreased.

\section{Glycophorin C/TdT-Positive Cells}

Ten cases (15\%) showed the presence of positive nonneoplastic cells after double labelling with glycophorin C and TdT (fig. 2e). Glycophorin C was not found to be ex-

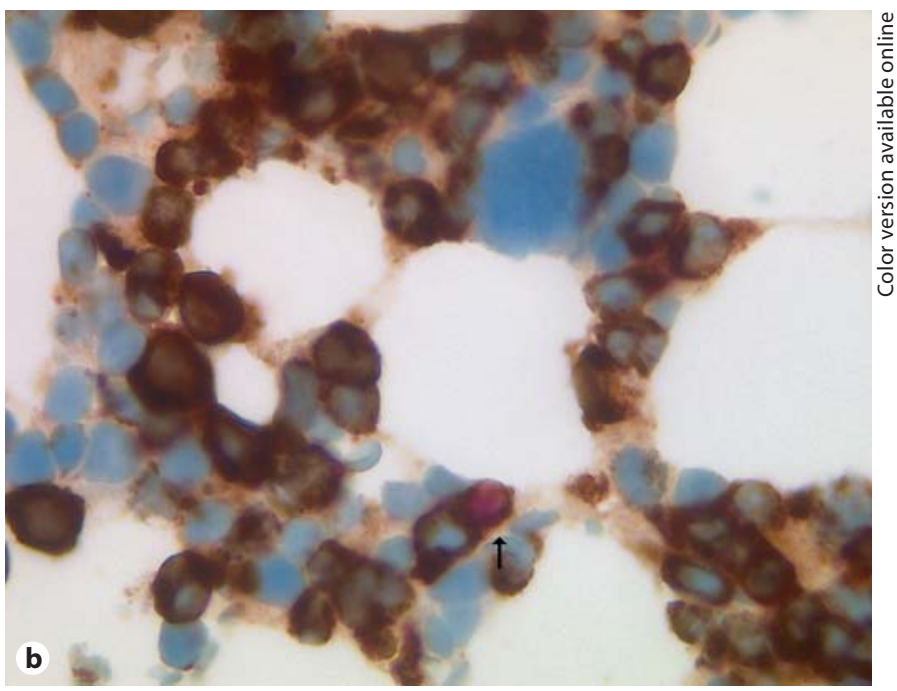

plasmic/membrane stain) co-expressing cell (arrow) has a smaller nucleus than the neoplastic population. b Acute promyelocytic leukaemia with a TdT( brown nuclear stain) and myeloperoxidase (red cytoplasmic stain) expressing cell (arrow) that exhibits a smaller nucleus than the TdT-negative neoplastic cells.

pressed by the AML cells in any of these cases. In 1 case after chemotherapy and in 2 cases after SCT the number of these cells increased, whereas in 1 case after SCT and 3 cases after chemotherapy these cells became undetectable. In most of these cases the number of double-positive cells was low ( $<30 \%$ of all TdT-expressing cells). In the single case with a higher ratio ( $70 \%$ of all TdT-positive cells), a relapse of AML was diagnosed and additionally verified by the haematologists after flow cytometry analysis.

\section{CD3/TdT-Positive Cells}

We found only 1 AML case which did not express lymphoid antigens harbouring a relatively high proportion of CD3/TdT-positive cells (30\%). The biopsy was made after first induction phase (fig. 2f). The number of these cells was reduced after the second chemotherapy.

\section{Patients with Relapse}

Five patients got a relapse during our study. In 3 of these cases a second biopsy showed elevated cells with CD117/TdT positivity, identified as tumour cells, 1 of them with additionally glycophorinC/TdT doublestained cells. The other 2 cases did not exhibit any double-stained positive cells. The most relevant data of the cases harbouring non-neoplastic TdT-positive cells other than haematogones are summarized in table 1. 

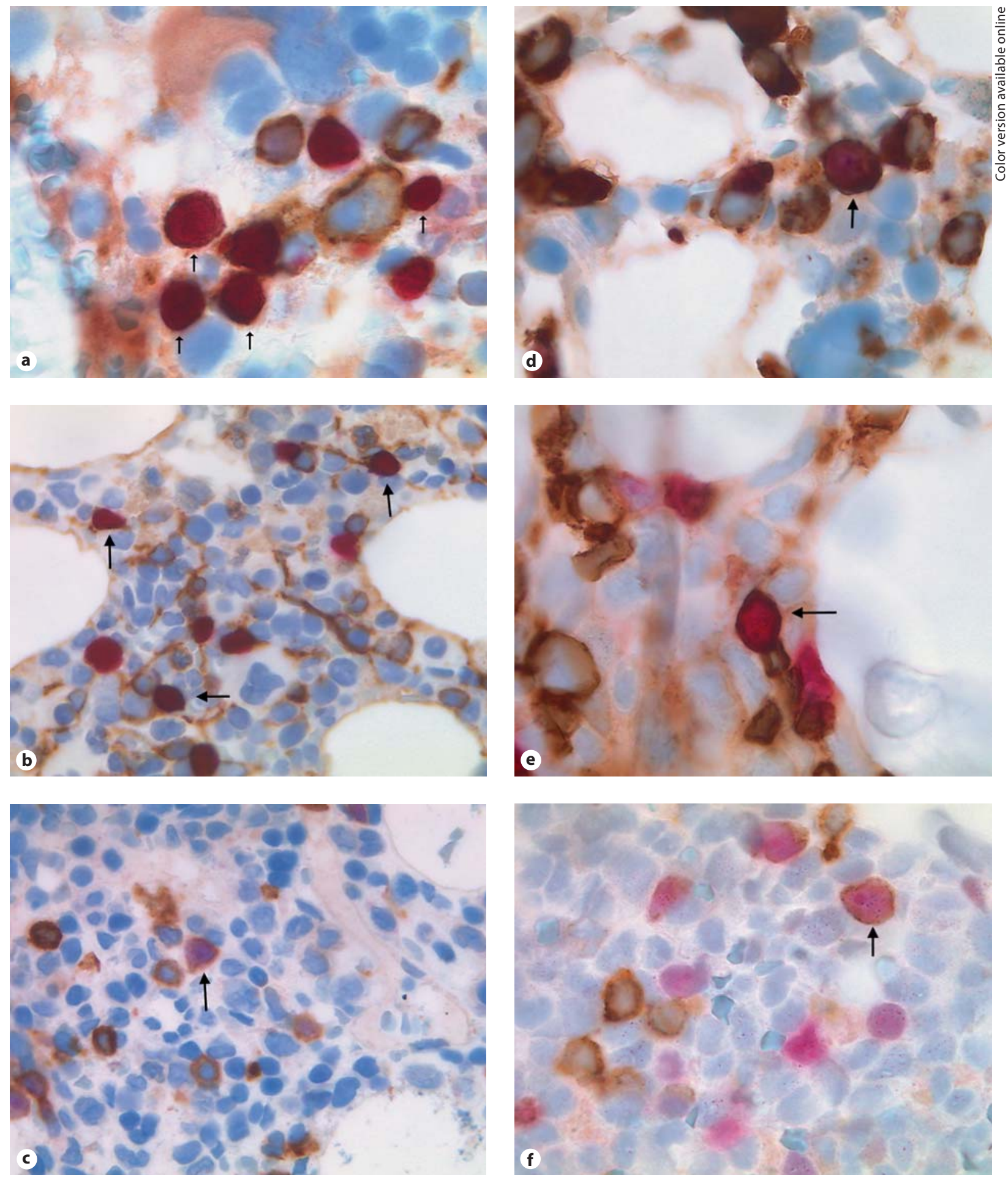

Fig. 2. Double labelling performed on decalcified paraffin embedded bone marrow trephines of AML patients. Haematogones were identified by the co-expression of TdT and CD19 (a) or CD10 (b). In addition to the TdT-expressing AML cells, 13\% of the cases contained non-neoplastic cells co-expressing CD117 and TdT (c). In $16 \%$ of the cases non-neoplastic cells co-expressing TdT and myeloperoxidase were identified (d). TdT-positive non-neoplastic cells co-expressing glycophorin $\mathrm{C}$ were found in $15 \%$ of the cases (e). One AML patient showed the presence of CD3 and TdT coexpressing cells after first induction therapy (f). Double labelling was performed in an automated immunostainer (Bond-maX ${ }^{\mathrm{TM}}$ ) using a combination of peroxidase (brown nuclear staining) and alkaline phosphatase (red cytoplasmic/membrane staining) detection systems. 
Table 1. Summary of the data on AML patients harbouring TdT-positive cells not assignable to haematogones or neoplastic cells

\begin{tabular}{|c|c|c|c|c|c|c|c|}
\hline Patient & Gender & Age & Diagnosis & $\begin{array}{l}\text { TdT/CD117 } \\
\text { co-expression }\end{array}$ & $\begin{array}{l}\text { TdT/glycophorin C } \\
\text { co-expression }\end{array}$ & $\begin{array}{l}\text { TdT/MPO } \\
\text { co-expression }\end{array}$ & $\begin{array}{l}\text { Follow-up } \\
\text { months }\end{array}$ \\
\hline 2 & $\mathrm{~F}$ & 56 & secondary AML & $20 \%$ & $10 \%$ & $1 \%$ & 17 \\
\hline $3 a$ & M & 77 & AML with maturation/M2 & $40 \%$ & 0 & $10 \%$ & 11 \\
\hline $3 b$ & & & & $1 \%$ & $30 \%$ & 0 & 14 \\
\hline 5 & $\mathrm{~F}$ & 20 & acute monocytic leukaemia/M5 & 0 & 0 & $10 \%$ & 16 \\
\hline 8 & $\mathrm{~F}$ & 48 & AML with maturation/M2 & $5 \%$ & 0 & $20 \%$ & 22 \\
\hline 9 & M & 60 & secondary AML & $80 \%$ & 0 & 0 & 13 \\
\hline 10 & M & 56 & acute monocytic leukaemia/M5 & $60 \%$ & 0 & 0 & 11 \\
\hline 13 & M & 63 & AML with minimal differentiation/M0 & $20 \%$ & 0 & 0 & 23 \\
\hline 14 & $\mathrm{~F}$ & 45 & acute monocytic leukaemia/M5 & 0 & 0 & $10 \%$ & 15 \\
\hline 18 & M & 47 & acute erythroleukaemia/M6 & 0 & 0 & $10 \%$ & 25 \\
\hline 20 & M & 57 & AML with maturation/M2 & $30 \%$ & 0 & 0 & 24 \\
\hline 21 & $\mathrm{~F}$ & 48 & AML with minimal differentiation/M0 & 0 & $10-20 \%$ & $10 \%$ & 23 \\
\hline 23 & M & 21 & acute myelomonocytic leukaemia/M4 & 0 & 0 & $10 \%$ & 14 \\
\hline 29 & $\mathrm{~F}$ & 72 & acute promyelocytic leukaemia/M3 & $20 \%$ & 0 & $20 \%$ & 13 \\
\hline 33 & M & 68 & AML with maturation/M2 & $70 \%$ & $1 \%$ & 0 & 21 \\
\hline 35 & $\mathrm{~F}$ & 67 & secondary AML & 0 & $5 \%$ & 0 & 23 \\
\hline 37 & M & 54 & AML with maturation/M2 & $1 \%$ & $10 \%$ & 0 & 13 \\
\hline 40 & M & 62 & AML without maturation/M1 & $5 \%$ & $5 \%$ & 0 & 16 \\
\hline 41 & M & 62 & acute myelomonocytic leukaemia/M4 & 0 & $10 \%$ & 0 & 22 \\
\hline 44 & $\mathrm{~F}$ & 52 & AML without maturation/M1 & $5 \%$ & $10 \%$ & 0 & 26 \\
\hline 53 & M & 61 & AML without maturation/M1 & 0 & 0 & $30 \%$ & 36 \\
\hline 57 & M & 32 & secondary AML & $70 \%$ & 0 & $5 \%$ & 23 \\
\hline 60 & $\mathrm{~F}$ & 64 & AML with maturation/M2 & 0 & $10 \%$ & $10 \%$ & 25 \\
\hline 64 & M & 65 & AML without maturation/M1 & 0 & $10 \%$ & $1 \%$ & 27 \\
\hline
\end{tabular}

Twelve patients harboured more than one TdT-positive population without features of neoplastic cells or haematogones. Interestingly, case 3 showed different immunophenotypes of the TdT-positive reactive cells in sequential biopsies.

\section{Discussion}

TdT-positive cells in bone marrow biopsies have been the topic of several investigations. Some authors have investigated TdT-positive cells in bone marrow biopsies from patients with acute leukaemia focussing on the separation of haematogones from tumour cells of ALL [11]. According to their results, TdT-positive cells were defined as haematogones (normal precursor B cells), which reflect the vigorous $B$ cell generation after treatment [10]. Other authors described primitive lymphoid progenitors in bone marrows with a $\mathrm{T}$ lineage reconstruction potential [12]. Regarding AML, only the tumour cells have been investigated correlating the expression pattern of different myeloid and lymphoid molecules and the various subtypes/disease entities [13]. None of the authors however investigated the antigen expression patterns of reactive TdT-positive cells.

This is the first study which systematically analyzed TdT-positive non-tumour cells in bone marrows of AML patients at first manifestation as well as after chemotherapy or SCT. To clarify the origin of these cells following lineage specific antibodies were chosen for double immunostaining: MPO, glycophorin C, CD19 and CD3. Additionally, the co-expression of TdT/CD10 and TdT/ CD117 was investigated. Considering the fact that patients with AML before and after therapy underlie a strong bone marrow suppression resulting in pancytopenia, we tried to find out whether TdT-positive cells represent a heterogeneous group of myeloid and lymphoid progenitor cells or not.

Our results regarding co-expression of TdT/CD10 as well as of TdT /CD19 are in line with the published data: in 40 and $48 \%$ of the cases, CD19- and CD10-positive cells co-expressed TdT, respectively [8]. Twenty-two cases harboured both subpopulations, so we concluded that these cells represented haematogones. Three cases showed CD10/TdT-positive cells as well as MPO/TdT-positive cells but no CD19/TdT-positive cells. As it is known that CD10 is also detectable in granulopoietic cells, the precise 
differentiation between haematogones and immature myeloid cells has not been possible in these cases. Our results with respect to TdT-positive cells with co-expression of molecules specific for erythropoiesis and granulopoiesis are surprising. In 18\% of all investigated cases we could identify a subpopulation of TdT-positive nonneoplastic cells (up to 30\%) co-expressing MPO, a lineage-specific molecule of granulopoietic cells. Similar results could be obtained regarding the double immunostaining with glycophorin C and TdT. In $15 \%$ of the cases we could identify TdT-positive non-tumour cells expressing this erythroid lineage-specific molecule (the relapse patient not added). Excluding the TdT-positive tumour cells in patients with first manifestation or in relapse, cells with double expression of CD117 and TdT were found in 9 cases (13\%). As all of these patients who showed cells with lineage-specific double expression with $\mathrm{TdT}$ are in clinical remission (as evidenced by flow cytometric analysis), these cells were interpreted as being non-neoplastic. Finally, 1 case was identified containing large numbers of TdT-positive cells co-expressing CD3 after the first induction therapy.

Another aspect of our study was to find out if the TdTpositive cells are increased or decreased after therapy: we expected an increase in TdT-positive non-neoplastic cells after treatment, because of the therapy-associated bone marrow depletion with the resulting need for regeneration [10]. Surprisingly, a decrease in these cells after ther- apy was detected at similar frequency as an increase. We propose that the high neoplastic cell load occupying 80 $100 \%$ of the total bone marrow cellularity during primary manifestation of AML has also resulted in a significant depression of haematopoiesis. Therefore, $41 \%$ of these marrows already showed an elevated TdT-positive nonneoplastic subpopulation before treatment. Finally, no differences in the increase or decrease of TdT-positive cells between patients treated either with chemotherapy or SCT could be observed.

Taken together this study could point out two important and new facts. First, double immunolabellings with TdT and different line-specific molecules (glycophorin C, CD3, CD19 and MPO) could demonstrate that nonneoplastic TdT-positive cells in bone marrow trephines are not always haematogones but show also features of myeloid origin. The second interesting point is that there were no differences in the number of TdT-positive cells before and after chemotherapy or SCT. In order to clarify why a small portion of myeloid cells is able to express TdT, molecular studies at single cell level are required.

\section{Acknowledgements}

The authors would like to thank F. Gocht, A. Lehmann and $\mathrm{H}$. Noyan for their excellent technical assistance.

\section{References}

1 Yang-Feng TL, Landau NR, Baltimore D, Francke U: The terminal deoxynucleotidyltransferase gene is located on human chromosome 10 (10q23-q24) and on mouse chromosome 19. Cytogenet Cell Genet 1986;43: 121-126.

-2 Landau NR, St John TP, Weissman IL, Wolf SC, Silverstone AE, Baltimore D: Cloning of terminal transferase cDNA by antibody screening. Proc Natl Acad Sci USA 1984;81: $5836-5840$

-3 Marshall AJ, Doyen N, Bentolila LA, Paige CJ, Wu GE: Terminal deoxynucleotidyl transferase expression during neonatal life alters $\mathrm{D}(\mathrm{H})$ reading frame usage and Ig-receptor-dependent selection of $\mathrm{V}$ regions. J Immunol 1998;161:6657-6663.

-4 Foa R, Casorati G, Giubellino MC, et al: Rearrangements of immunoglobulin and $\mathrm{T}$ cell receptor $\beta$ and $\gamma$ genes are associated with terminal deoxynucleotidyl transferase expression in acute myeloid leukemia. J Exp Med 1987;165:879-890.
5 Zheng J, Wang X, Hu Y, et al: A correlation study of immunophenotypic, cytogenetic, and clinical features of $180 \mathrm{AML}$ patients in China. Cytometry B Clin Cytom 2008;74: 25-29.

6 Longacre TA, Foucar K, Crago S, et al: Hematogones: a multiparameter analysis of bone marrow precursor cells. Blood 1989;73: 543-552.

7 Kahng J, Shin SY, Han K: Proportions of cells expressing CD38-/CD34+, CD38+/CD34+, CD19+/CD34+, or CD13,33+/CD34+ in the regenerating bone marrows during complete remission of acute leukemia or after bone marrow transplantation. Korean J. Lab Med 2007;27:406-413.

8 Muehleck SD, McKenna RW, Gale PF, Brunning RD: Terminal deoxynucleotidyl transferase (TdT)-positive cells in bone marrow in the absence of hematologic malignancy. Am J Clin Pathol 1983;79:277-284.

-9 Rosse C, Kraemer MJ, Dillon TL, McFarland $\mathrm{R}$, Smith NJ: Bone marrow cell populations of normal infants; the predominance of lymphocytes. J Lab Clin Med 1977;89:1225-1240.
10 Wolf E, Harms H, Winkler J, et al: Terminal deoxynucleotidyl transferase-positive cells in trephine biopsies following bone marrow or peripheral stem cell transplantation reflect vigorous B-cell generation. Histopathology 2005;46:442-450.

11 Hurford MT, Altman AJ, DiGiuseppe JA, Sherburne BJ, Rezuke WN: Unique pattern of nuclear TdT immunofluorescence distinguishes normal precursor B cells (hematogones) from lymphoblasts of precursor Blymphoblastic leukemia. Am J Clin Pathol 2008;129:700-705.

12 Perry SS, Welner RS, Kouro T, Kincade PW, Sun XH: Primitive lymphoid progenitors in bone marrow with $\mathrm{T}$ lineage reconstituting potential. J Immunol 2006;177:2880-2887.

13 Drexler HG, Sperling C, Ludwig WD: Terminal deoxynucleotidyl transferase (TdT) expression in acute myeloid leukemia. Leukemia 1993;7:1142-1150. 